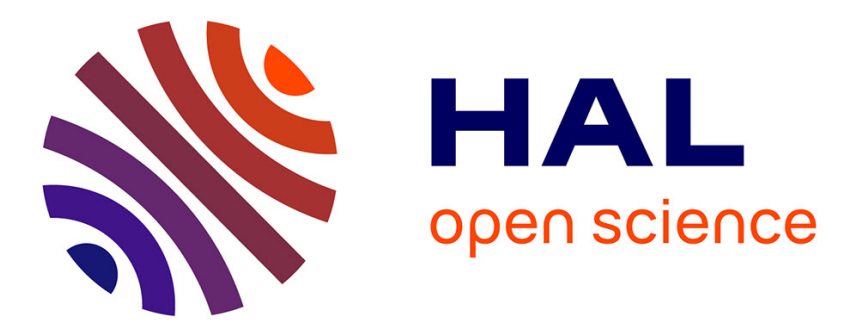

\title{
Dynamics of plastic deformation based on restoring and energy dissipative mechanisms in plasticity
}

\author{
S Yoshida
}

\section{To cite this version:}

S Yoshida. Dynamics of plastic deformation based on restoring and energy dissipative mechanisms in plasticity. Physical Mesomechanics, 2008, 11 (3-4), pp.137-143. hal-01092050

\section{HAL Id: hal-01092050 \\ https://hal.science/hal-01092050}

Submitted on 8 Dec 2014

HAL is a multi-disciplinary open access archive for the deposit and dissemination of scientific research documents, whether they are published or not. The documents may come from teaching and research institutions in France or abroad, or from public or private research centers.
L'archive ouverte pluridisciplinaire HAL, est destinée au dépôt et à la diffusion de documents scientifiques de niveau recherche, publiés ou non, émanant des établissements d'enseignement et de recherche français ou étrangers, des laboratoires publics ou privés. 


\title{
Dynamics of plastic deformation based on restoring and energy dissipative mechanisms in plasticity
}

\author{
S. Yoshida \\ Southeastern Louisiana University, Hammond, Louisiana 70402, USA
}

\begin{abstract}
The dynamics of plasticity is considered based on the field theoretical approach developed by physical mesomechanics. The equation of motion governing mesoscopic volume elements in plastically deforming media is derived from the mesomechanical field equation. Theoretical analysis on this equation of motion indicates that in the plastic regime solid-state media exert two types of forces; the restoring force and energy dissipating force. The former is associated with the shear modulus, and causes the displacement field to be oscillatory. The latter is associated with a quantity analogous to the electric charge, and causes the displacement field to be decaying. Experimental observations that support these theoretical considerations are presented.
\end{abstract}

\section{Introduction}

The invention of physical mesomechanics [1] has introduced a number of new ideas in the study of plastic deformation. Among those, the concept of rotational mode of deformation [2] is of particular importance in understanding of the dynamics of plastic deformation at the fundamental level of physics. By considering finite volumes in continuous media, physical mesomechanics has successfully incorporated the elegance of the linear algebraic description of the elastic theory [3] into the complexity of anisotropy in plasticity. When defects are generated in a continuous medium, physical mesomechanics explains, the medium is broken into structural units bounded by those defects where each of these units individually gains rotational degrees of freedom [4]. Consequently, different parts of the object rotate differently, i.e., the object experiences rotational deformation. This situation is contrastive to elasticity where rotational displacement represents rigid body motion, not deformation. In the linear algebraic terms, the rotation matrix element is a function of the spatial coordinates, which in elasticity is a constant.

From the gauge theoretical point of view, the coordinate dependence of the rotational matrix element can be interpreted as that the rotational dynamics is local, as opposed to global in elasticity. Similarly, it follows that in plasticity

Corresponding author

Prof. Sanichiro Yoshida, e-mail: syoshida@selu.edu the distortion matrix elements (the normal and shear strain) have coordinate dependences $[2,5]$. Since these matrix elements contain spatial differentiation, this necessitates the replacement of the partial derivatives with covariant derivatives, i.e., the introduction of a gauge. Using linear transformation of the $\operatorname{GL}(3, R)$ group and finding a Lagrangian invariant under the transformation, Egorushkin [6] derived the field equation and formulated the spatiotemporal behavior of dislocations as wave dynamics $[4,6]$. Formulaically, this field equation is analogous to Maxwell equation of electrodynamics [7]. The wave nature of plastic deformation has also been experimentally verified at the macroscopic level; e.g., Danilov et al. [8,9] observed strain waves in various metal specimen, and Yoshida et al. [10] observed displacement waves in aluminum alloys.

Essentially, this wave dynamics represents the synergetic interaction between the translational and rotational modes of deformation. An interesting way of viewing this synergetic effect is to interpret it as a self-stabilizing mechanism analogous to Faraday-Lenz's law of electrodynamics [5]. If an external agent causes a change in magnetic field over time, the electric field induces a scalar potential so that the change in the magnetic field is compensated. A similar mechanism operates in plastically deforming media via a restoring force [11]. In the elastic regime, media posses the restoring force known as the elastic force whose magnitude is proportional to the displacement from the translational equilibrium. In the plastic regime, media lose the elastic force, but instead, 
gain restoring torque whose magnitude is proportional to the rotation from the rotational equilibrium. The driving force of this restoring torque is shear stress. Like the electrodynamics case, this mechanism leads to the oscillatory characteristics of the field variables, which propagates through the media as transverse waves. In the plastic regime, there is additional force, which causes energy dissipation. This force represents a longitudinal effect similar to the electric force, as opposed to the transverse effect of the restoring torque. The aim of this paper is to consider these two forces, called the plastic force comprehensively, and the associated effect that appear in the plastic regime. The mesomechanical field equation is viewed as the equation of motion that governs the dynamics associated with the plastic force. The energy dissipating force is viewed as a field force acting on a quantity analogous to the electric charge (called deformation charge). The physical meanings of the charge and associated energy dissipation process is discussed. Experimental observations that support these discussions are presented.

\section{Theoretical consideration}

\subsection{Field equation and equation of motion}

The restoring and energy dissipating natures of the plastic force can be conveniently explained with the help of mesomechanical field equation. After the summation over the group indices, the field equation can be put in the following form $[2,11]$.

$$
\begin{aligned}
& \nabla \cdot \mathbf{v}=j^{0} \\
& \nabla \times \mathbf{v}=\frac{\partial \boldsymbol{\omega}}{\partial t},
\end{aligned}
$$
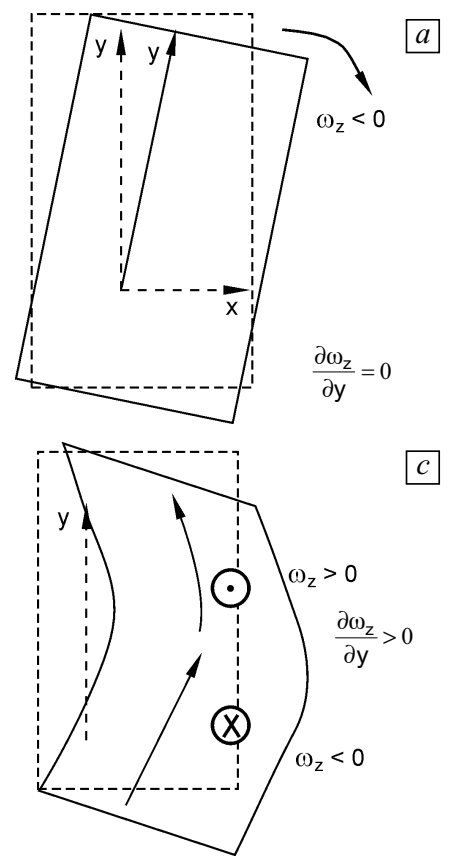

$$
\begin{aligned}
& \nabla \times \boldsymbol{\omega}=-\frac{1}{c^{2}} \frac{\partial \mathbf{v}}{\partial t}-\mathbf{j}, \\
& \nabla \times \boldsymbol{\omega}=0,
\end{aligned}
$$

where $j^{0}$ and $\mathbf{j}$ are the time and spatial components of the four vector associated with the symmetry charge, $\mathbf{v}$ and $\boldsymbol{\omega}$ are the field variables representing the translational and rotational displacement of the deformation field, and $c$ is the phase velocity of the wave characteristics of the field variables. Eq. (3) can be interpreted as the equation of motion representing the dynamics of a volume element (with a unit volume) in a plastically deforming medium. Rearrangement of the terms as below indicates its meaning explicitly,

$$
\varepsilon \frac{\partial \mathbf{v}}{\partial t}=-\frac{1}{\mu} \nabla \times \boldsymbol{\omega}-\frac{\mathbf{j}}{\mu} .
$$

In Eq. (5) the phase velocity $c$ has been replaced with $\sqrt{1 / \varepsilon \mu}$, where $\varepsilon$ is the density and $1 / \mu$ is the shear modulus of the medium, as explained below. The left-hand side of Eq. (5) represents the product of the mass and acceleration of the unit volume element, and the right-hand side represents the plastic force as the external forces acting on the volume element; the first term represents the restoring force per unit volume, and the second term the energy dissipating force per unit volume.

\subsection{Restoring force in plasticity}

To further investigate the restoring force, multiply the volume $\mathrm{d} x \mathrm{~d} y \mathrm{~d} z$ to both-hand side of Eq. (5) and consider the $x$ component of the first term on the right-hand side. Fig. 1 illustrates the situation schematically, where the $x$ component of the restoring force is related to the $y$ depen-

Fig. 1. Plastic restoring force due to differential rotation

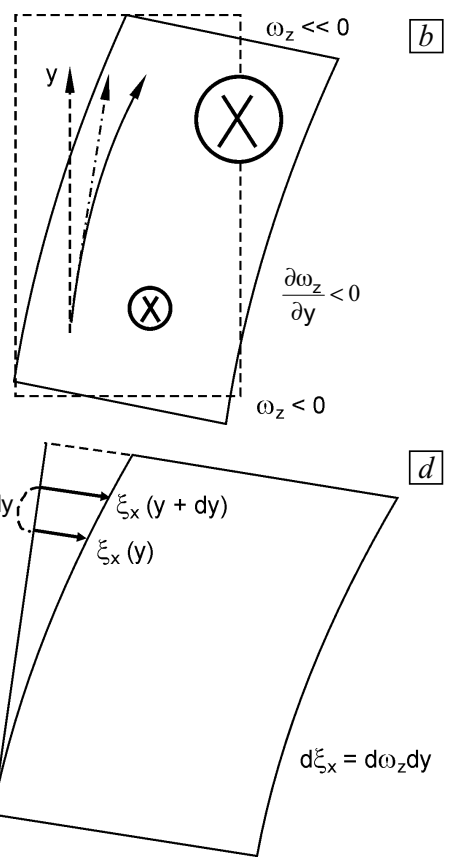


dence of the $z$ component of rotation vector $\omega$. The $y$ component of the force can be argued in the same fashion via the $x$ dependence of $\omega_{z}$ :

$$
f_{x}^{\text {restore }}=-(\nabla \times \boldsymbol{\omega})_{x} \frac{1}{\mu} \mathrm{d} x \mathrm{~d} y \mathrm{~d} z=-\frac{\partial \omega_{z}}{\partial y} \frac{1}{\mu} \mathrm{d} x \mathrm{~d} y \mathrm{~d} z .
$$

Figure 1 $(a)$ illustrates the situation where rotation does not depend on $y$, i.e., $\partial \omega_{z} / \partial y=0$. This situation represents the elastic deformation where rotation is not deformation and the plastic restoring force does not exist. Figure $1(b)$ illustrates the situation where the clockwise rotation increases as going up in the positive $y$ direction, i.e., $\partial \omega_{z} / \partial y<0$. Accordingly, the restoring force proportional to $-(\nabla \times \boldsymbol{\omega})_{x}=-\partial \omega_{z} / \partial y$ is positive, or rightward. Finally, Figure 1(c) illustrates the situation where the rotation is clockwise in the lower part and counterclockwise in the upper part, i.e., $\partial \omega_{z} / \partial y>0$. The restoring force is negative or leftward.

$\sqrt{1 / \mu}$ can be identified as representing the shear modulus as follows. As Figure 1 $(d)$ illustrates, the line connecting the lateral equilibrium points (denoted by the $y$-axis) rotates as the medium experiences the rigid body rotation. Consequently, when $\partial \omega_{z} / \partial y \neq 0$, the displacement from the lateral equilibrium point varies as a function of $y$, and the differential displacement between $y$ and $y+\mathrm{d} y$ is given by $\mathrm{d} \xi_{x}=\mathrm{d} \omega_{z} \mathrm{~d} y$. Replacing $\partial \omega_{z} / \partial y \mathrm{~d} y$ with $\mathrm{d} \omega_{z}$, dividing both-hand side by $\mathrm{d} x \mathrm{~d} z$, and replacing $f_{x}^{\text {restore }} / \mathrm{d} x \mathrm{~d} z$ with the shear stress $\sigma_{x}$ and $\mathrm{d} \omega_{z}=\mathrm{d} \xi_{x} / \mathrm{d} y$ with the shear strain $\tau_{x}$, Eq. (6) can be rewritten as

$$
\sigma_{x}=-\tau_{x} \frac{1}{\mu} \text {. }
$$

Equation (7) indicates that $1 / \mu$ represents the ratio $\sigma_{x} / \tau_{x}$, i.e., the shear modulus.

With this interpretation, $\sqrt{1 / \varepsilon \mu}$ has a unit of

$$
\sqrt{\left(\mathrm{m}^{3} / \mathrm{kg}\right)\left(\mathrm{N} / \mathrm{m}^{2}\right)}=\mathrm{m} / \mathrm{s} \text {, }
$$

which rationalizes the above interpretation that $\sqrt{1 / \varepsilon \mu}$ represents the phase velocity. Thus the first term on the righthand side of Eq. (5) represents the oscillatory dynamics based on the rotational recovery force, which travels at the phase velocity $\sqrt{1 / \varepsilon \mu}$. The negative sign indicates that the force is restoring.

\subsection{Energy dissipating force}

The second term on the right-hand side of Eq. (5) can be interpreted as representing an energy dissipating mechanism analogous to electric conduction. When an electric field is applied to an electrically conductive medium, electric force acts on free charges. Consequently, the charges drift through the medium and the energy stored in the field is dissipated. A similar mechanism exists in plasticity. To understand this mechanism, below we first consider a quantity equivalent to the electric charge and then its flow.

Consider in Fig. 2 that an infinitesimally small volume element displaces in the direction of $\xi$ in time $\mathrm{d} t$, and consequently, its velocity changes by $\mathrm{d} v_{\xi}$,

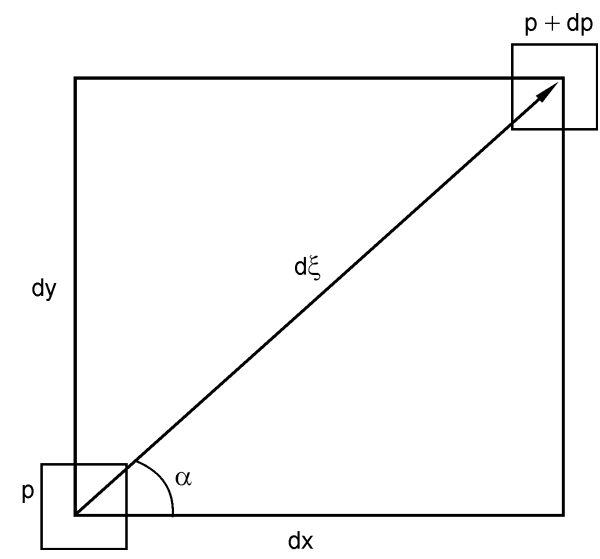

Fig. 2. Volume element experiences a change in momentum

$$
\mathrm{d} \mathbf{v}_{\xi}=\frac{\partial \mathbf{v}_{\xi}}{\partial \xi} \mathrm{d} \xi
$$

The resultant change in momentum of this volume element over $\mathrm{d} t$ is given as

$$
\frac{\mathrm{d} \mathbf{p}}{\mathrm{d} t}=m \frac{\mathrm{d} \mathbf{v}_{\xi}}{\mathrm{d} t}=m \frac{\partial v_{\xi}}{\partial \xi} \frac{\mathrm{d} \xi}{\mathrm{d} t} \mathbf{e}_{\xi}=m \frac{\partial v_{\xi}}{\partial \xi} \mathbf{v}_{\xi},
$$

where $\mathbf{e}_{\xi}$ is the unit vector along the $\boldsymbol{\xi}$ axis. Suppose $\mathbf{v}_{\boldsymbol{\xi}}$ is in the first quadrant of the $x-y$ plane with angle $\alpha$ to the $x$ axis. With $\mathrm{d} x=\mathrm{d} \xi \cos \alpha$ and $\mathrm{d} y=\mathrm{d} \xi \sin \alpha, \mathrm{d} v_{\xi} / \mathrm{d} \xi$ can be rewritten as

$$
\begin{aligned}
\frac{\mathrm{d} v_{\xi}}{\mathrm{d} \xi} & =\frac{\partial v_{\xi}}{\partial x} \frac{\mathrm{d} x}{\mathrm{~d} \xi}+\frac{\partial v_{\xi}}{\partial y} \frac{\mathrm{d} y}{\mathrm{~d} \xi}= \\
& =\frac{\partial v_{\xi}}{\partial x} \cos \alpha+\frac{\partial v_{\xi}}{\partial y} \sin \alpha=\frac{\partial u}{\partial x}+\frac{\partial v}{\partial y},
\end{aligned}
$$

where, $u=v_{\xi} \cos \alpha$ and $v=v_{\xi} \sin \alpha$ are the $x$ and $y$ component of $\mathbf{v}_{\xi}$. With these expressions, $\mathrm{d} \mathbf{p} / \mathrm{d} t$ in the $x-y$ coordinate becomes

$$
\frac{\mathrm{d} \mathbf{p}}{\mathrm{d} t}=m\left(\frac{\partial u}{\partial x}+\frac{\partial v}{\partial y}\right) \mathbf{v}_{\xi} .
$$

Eq. (11) indicates that when the volume element moves in the flow field with the velocity variation $\mathrm{d} v_{\xi} / \mathrm{d} \xi$ in $\mathrm{d} t$, the surrounding part of the medium exerts force so that the volume element's momentum changes, i.e., $f=\mathrm{d} p / \mathrm{d} t$. The quantity $(\partial u / \partial x+\partial v / \partial y)$ appearing on the right-hand side of Eq. (11) can be interpreted as the two-dimensional representation of $\nabla \cdot \mathbf{v}$ that appears on the left-hand side of Eq. (1). The right-hand side of this equation can be rewritten as $\rho=j^{0} / \varepsilon$, where $j^{0}$ is the charge of symmetry [2,11], $\varepsilon$ is the density of the medium, and $\rho$ is a quantity equivalent to the electric charge density. Therefore, the force exerted by the surrounding part of the volume element can be viewed as the field force acting on the charge $q=m \nabla \cdot \mathbf{v}$ of the volume element, where $m$ denotes the total mass of the volume element. In this view, a positive charge $(q>0)$ receives the field force in the same direction as $\mathbf{v}$ and a negative charge in the opposite direction. 
The above argument indicates that all infinitesimal volume elements inside the velocity variation $\mathrm{d} v_{\xi}=$ $=(\partial u / \partial x+\partial v / \partial y) \mathrm{d} \xi$ are subject to the field force proportional to the local velocity $\mathbf{v}_{\xi}$. Figure $3(a)$ illustrates a simplified picture where $\mathrm{d} v_{\xi}$ is represented by a smooth variation from the low velocity level to the high velocity level. The whole region of this velocity variation can be regarded as a positive charge. If the velocity field is steady, the charge remains at the same location. If, for some reason, the charge drifts in the field, the volume corresponding to the low velocity level increases and the volume corresponding to the high velocity level decreases; the total momentum of the medium decreases. Figure 3(b) illustrates the situation schematically. A possible cause for such a drift of the charge is that the point in the medium where the velocity variation rises (point $P$ in Fig. 3) becomes weaker than the rest of the medium, and therefore, the stretch of the entire medium is concentrated around this point. Consequently, the whole pattern of the velocity variation shifts rightward in a short period of time. Experiment indicates that such an abrupt shift of velocity-variation-pattern indeed occurs (see Section 3). Another experiment reveals that the abrupt shift is accompanied by acoustic emission, indicating that the concentrated stretch is caused by partial breakage of the medium [12]. Although Fig. 3 uses a simple case as an example, the same argument holds when the variation of the velocity is more complicated, or a negative charge drifts in the opposite direction to the flow $v$.

Combination of Eqs. (1) and (3) leads to an equation of continuity, which indicates the charge conservation and allows us to put the second term on the right-hand side of Eq. (5) in the form of $j / \mu=\rho W_{\mathrm{d}}$. Here $W_{\mathrm{d}}$ is the drift velocity of the charge. By interpreting that the drift is due to the above mentioned field force, this form of $j / \mu$ can be identified as the motion of the charge illustrated by Fig. 3 . Dimensional analysis indicates that $j / \mu$ is in $\left[\mathrm{N} / \mathrm{m}^{3}\right]$. It is therefore interpreted that $j / \mu$ represents the effective force corresponding to the momentum loss per unit volume. As a force proportional to velocity $W_{\mathrm{d}}$, this force is basically a velocity damping force; it is expected that in the plastic regime, the oscillatory characteristics of the displacement field decays exponentially. As discussed below (see Section 3.2), experiment shows that the displacement field under constant tensile loading indeed decays exponentially.

\subsection{Two-dimensional model of development of deformation}

Based on the dynamics explained above, the development of deformation from the elastic to plastic regime can be conveniently illustrated by a two-dimensional model. Consider a $x-y$ plane in a medium experiencing deformation under a tensile load, where the tensile force is in the positive $y$ direction, and the positive $z$ is in the direction out of the plane. Initially, the translational displacement $v$ induced by the tensile load is uniform over the whole sample. Under this condition, $\omega$, if exists, represents rigid body rotation. In other words, the whole plane is represented by the same $\omega$, and $\nabla \times \omega=0$. This situation represents the elastic regime of the deformation.

When the deformation reaches a certain level, a certain part of the medium becomes weaker than the other part. Consequently, the weaker part stretches more than the other part. In other words, the field of the translational displacement becomes non-uniform. This non-uniformity induces local rotational fields in the surrounding area. Figure 4 illustrates such a situation schematically. Suppose that all points in the plane shown in Fig. 4a initially displace uniformly in the vertical direction; the entire region experiences the same stretch. At a certain point of time, the central part of the plane becomes weaker, and consequently, point $A$ displaces more than point $A^{\prime}$ or $A^{\prime \prime}$, while point $B$ displaces less than point $B^{\prime}$ or $B^{\prime \prime}$. This induces local rotations in the four surrounding areas; the top-right and bottom-left rotates clockwise and the top-left and bottom-right counterclockwise. Thus the whole region is represented by non-zero $\nabla \times \boldsymbol{\omega}$, i.e., the restoring force in Eq. (5) has a finite value. This is the beginning of plastic deformation.

Now consider the boundaries of these four regions represented by the local rotations. In these boundaries, the medium tends to displace in the direction of $\nabla \times \boldsymbol{\omega}$; for example, at the boundary between the top-right and bottom-right regions the medium tends to displace to the left. In response to this displacement, the medium exerts restoring force in the opposite direction as represented by the first term on the right hand side of Eq. (5). In this context, the unit volume represented by $\varepsilon$ is located at the boundary of the local rotations.

The event that the central region becomes weaker can be modeled as the stiffness of the medium between point $A$
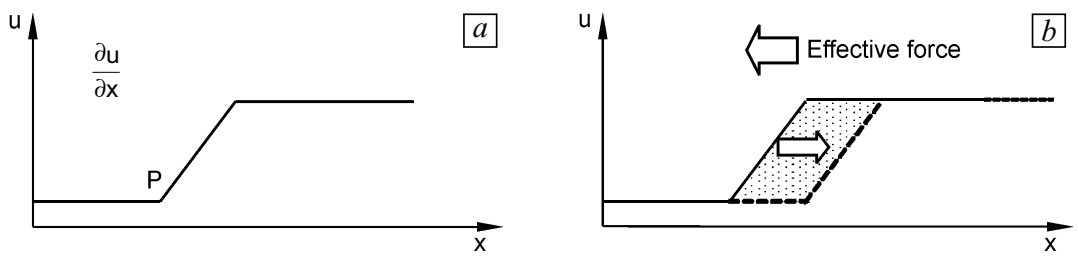

Fig. 3. A positive charge drifting in the flow direction 

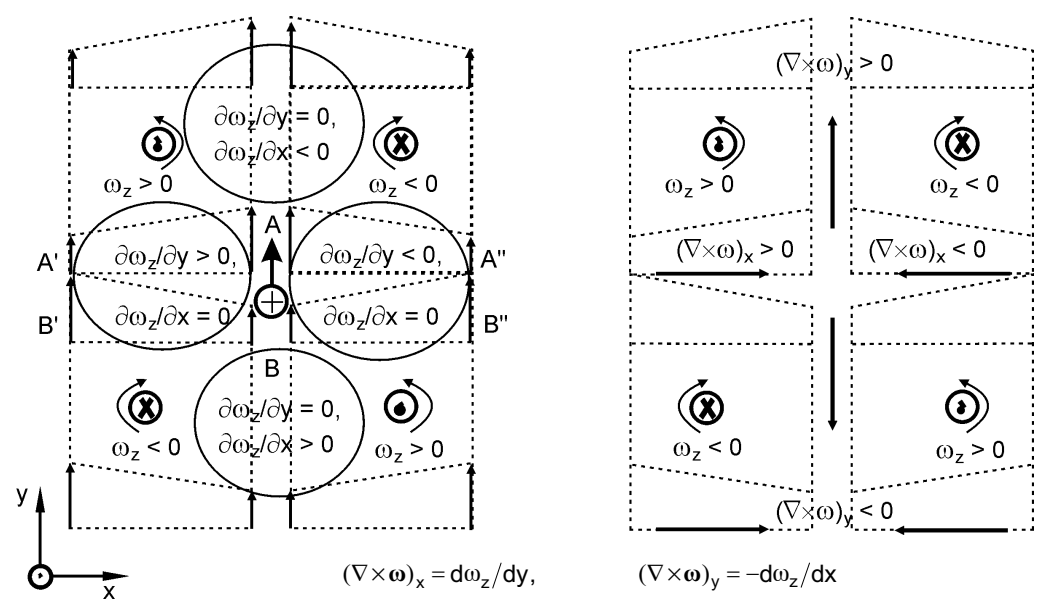

Fig. 4. Two-dimensional model of development of deformation

and $B$ suddenly decreases. Imagine that you are holding a mass connected to a stretched spring and the spring constant decreases suddenly. Because the decrease in the spring constant lowers the spring force and you keep exerting the same force, at the moment the spring constant decreases you and the other end of the spring will recoil in mutually opposite directions. Consequently, the region between point $A$ and $B$ stretches more than the surrounding regions, which can be represented by $\mathrm{d} v / \mathrm{d} y$ or locally concentrated positive charge density. In accordance with the second term on the right-hand side of Eq. (5), the velocity field exerts the field force on this charge, and consequently this region containing the positive charge is pushed in the direction of the local velocity at this point. This motion of the charge dissipates energy as mentioned above. Note that as the positive charge is pushed by the velocity field, the leading edge whose velocity is greater than the tailing edge (because $\mathrm{d} v / \mathrm{d} y>0)$ displaces more than the tailing edge. Consequently, the charge density decreases with the passage of the time. Thus after some time, the field force vanishes and the restoring force proportional to the elongation resumes. This causes the zig-zag shaped loading characteristics known as the serration.

\section{Experimental observations}

Experiments have been performed with ESPI (Electronic Speckle Pattern Interferometry) [13] to measure displacement on deforming samples under tensile loading. Figure 5 illustrates a typical ESPI setup used. The beam splitter splits the optical beam from the laser source into two interferometric paths that are recombined on the sample surface after expanded by the beam expanders. The interferometric image of the sample is captured by the CCD camera at a constant time step, and the digital image data is transferred to computer memory. Fringe patterns containing the displacement data are formed by subtracting the image data taken at a certain time step from that taken at another time step. The interferometric configuration shown in Fig. 5 is sensitive to horizontal displacement. A similar ESPI setup sensitive to vertical displacement was used for the vertical analysis.

\subsection{Shear-band as a developed charge}

Figure 6 shows a typical pair of fringe patterns representing the horizontal and vertical displacement. The dark stripe represents the region where the displacement is an integer multiple of the unit length corresponding to the phase change of $2 \pi$. Typically, the unit length is somewhere between $0.3-$ $0.5 \mu \mathrm{m}$. In many occasions, a bright band pattern like the one seen near the top of Fig. 6 is observed in a usual fringe pattern. As this figure indicates, the bright patterns are always observed at the same location in the horizontal and vertical fringe patterns corresponding to the same time. Toyooka et al. [14] discovered that this bright pattern actually consists of a number of concentrated parallel fringes. As reported in [15], the bright pattern is often observed near a Lüders band and propagates at the same speed. In a later stage of deformation, often this band pattern becomes stationary and the sample always fractures at that location [16]. The fringe patterns observed in the upper and lower

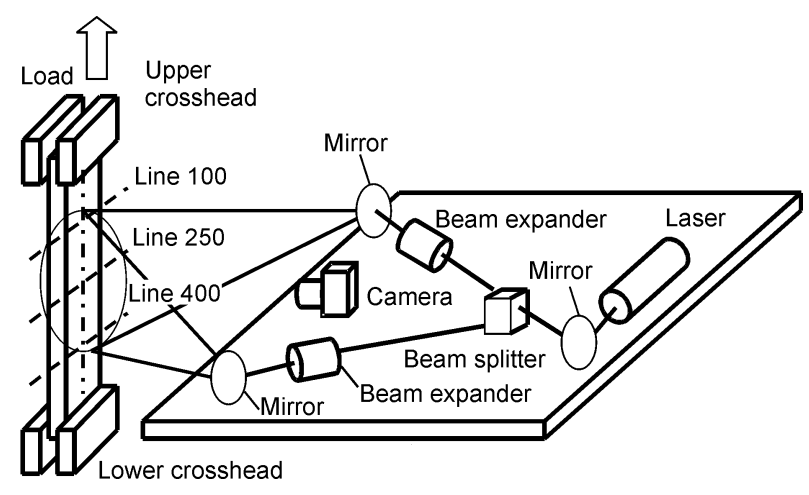

Fig. 5. ESPI setup for displacement measurement 


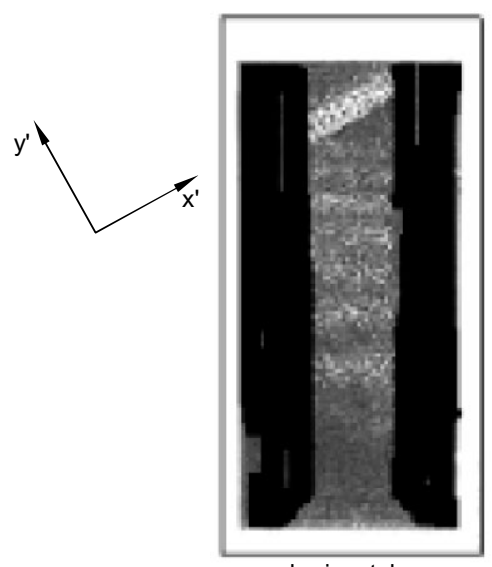

horizontal

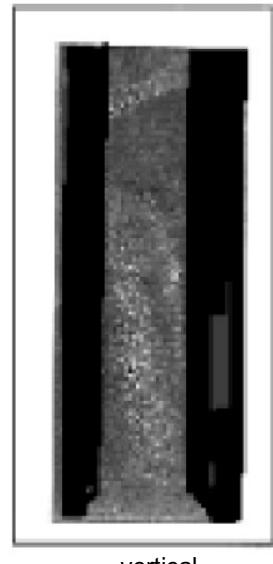

vertical
Fig. 6. Typical fringe patterns representing horizontal (left) and vertical (right) displacement

sides of this band pattern indicate that the sample experiences bodily rotations on the regions bounded by the band pattern. All these observations indicate that this band pattern represents stress concentration associated with localized shear deformation. Thus hereafter it is called the shear-band.

The observation that the parallel fringes in a shear-band run along a certain axis, say the $x^{\prime}$-axis in Fig. 6, both in the horizontal and vertical fringe patterns means that both the horizontal and vertical velocities ( $u$ and $v$ ) have no $x^{\prime}$ dependence or $\partial u / \partial x^{\prime}=\partial v / \partial x^{\prime}=0$. On the other hand, $\partial u / \partial y^{\prime}$ and $\partial v / \partial y^{\prime}$ are high values because the fringe density is high. In this situation, $\omega_{z}^{\prime}=-\partial u^{\prime} / \partial y^{\prime}$, where $z$ is normal to the $x^{\prime}-y^{\prime}$ plane in the right-hand system, and $u^{\prime}$ and $v^{\prime}$ are, respectively, the velocity components in the $x^{\prime}$ and $y^{\prime}$ direction. Therefore, $\left(\nabla \times \boldsymbol{\omega}^{\prime}\right)_{y^{\prime}}=-\partial \omega_{z}^{\prime} / \partial x^{\prime}=\partial^{2} u^{\prime} / \partial y^{\prime} \partial x^{\prime}=0$, while $\left(\nabla \times \boldsymbol{\omega}^{\prime}\right)_{x^{\prime}}=\partial \omega_{z}^{\prime} / \partial y^{\prime}=-\partial^{2} u^{\prime} / \partial y^{\prime 2}$ has a non-zero value; i.e., the recovery force represented by the first term on the right-hand side of Eq. (5) has only the component parallel to the shear-band like fringes. Figure 7 shows twodimensional velocity field at a certain time step (Fig. 7(a)) and a time step later (Fig. 7(b)), and the subtraction of the former from the latter (Fig. 7(c)). Since it is a change in velocity over time, Figure 7(c) represents the acceleration field. One time step after, the sample showed a shear-band
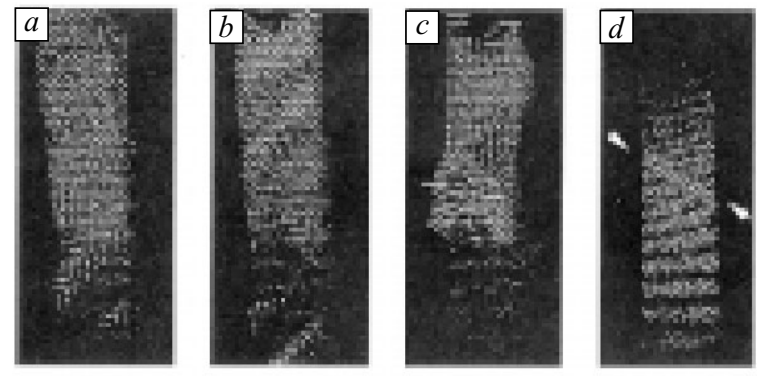

Fig. 7. Acceleration field near the shear-band like fringes along the boundary of the two vortex-like acceleration fields (Fig. 7(d)). Note that the acceleration vectors near the shearband are parallel to the band [17]. This observation clearly supports the above argument that the recovery force is parallel to the shear-band.

\subsection{Displacement wave}

This oscillatory characteristics of displacement fields have been observed in aluminum alloy plates under tensile loading with constant pulling speeds. Figure $8(a)$ shows the oscillation observed in the displacement vector component perpendicular to the tensile axis. The displacement data was obtained via analysis of fringe patterns formed with the same ESPI configuration as in Fig. 5. The plot shown in Fig. $8(a)$ is the decaying oscillation observed at the three reference points indicated in Fig. 5 (three points where the three horizontal lines cross the vertical line along the center of the sample). Figure $8(b)$ shows similar plot for a different sample. In this case, the displacement field clearly shows over-damping characteristics. Figure $8(c)$ is the case when the shear-band appears intermittently while the displace-
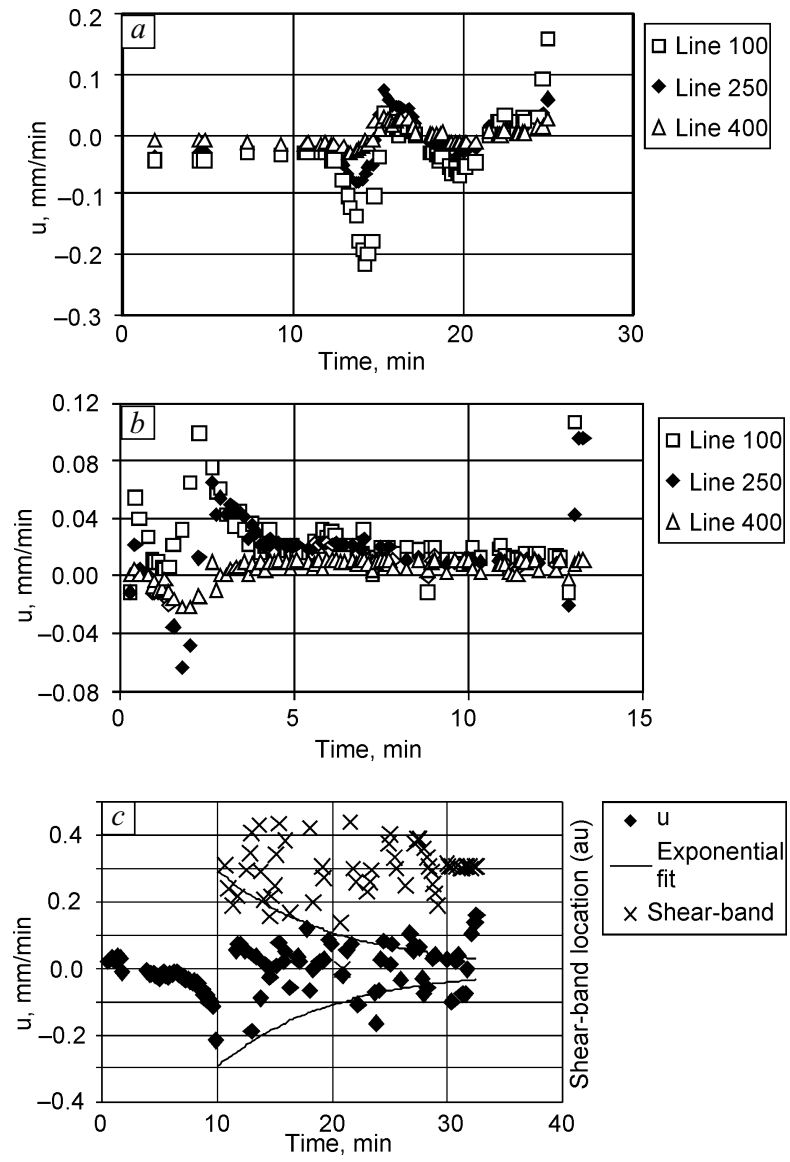

Fig. 8. Oscillation characteristics of displacement component perpendicular to tensile axis: decaying-oscillation $(a)$, over-damping $(b)$, with shear-bands $(c) . \times$ indicates the vertical locations on the sample where the shear-band appears intermittently (with an arbitrary unit) 


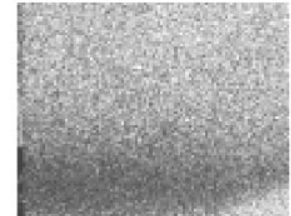

No. 2

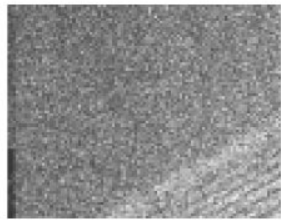

No. 32

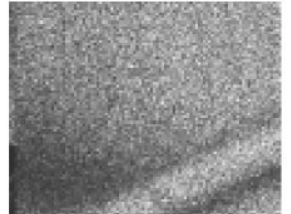

No. 44

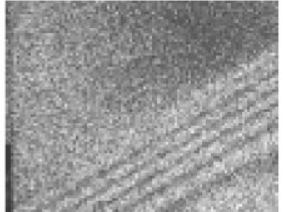

No. 68

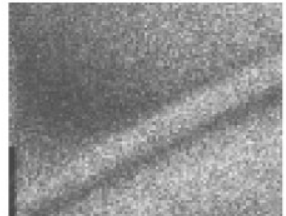

No. 87

Fig. 9. Periodic change in the density of concentrated fringes inside a shear band. The rightmost picture shows the whole image of the shear-band observed embedded in usual fringes. The numbers under enlarged pictures of the parallel fringes inside the shear-band indicate the frame numbers of the CCD camera

ment vector component shows oscillatory characteristics. The dashed line that connects the peaks of the oscillation indicates the exponential decay due to the energy dissipating force. It is seen that at the moment when the shear-band appears the oscillation is discontinuous.

The observation of discontinuity in the oscillatory characteristics can be explained by the above-argued restoring and energy dissipating mechanisms in plasticity. Figure 9 shows the whole image of a shear-band (the rightmost picture) and enlarged pictures of the concentrated parallel fringes inside the shear-band. The enlarged pictures were taken by a second CCD camera with a higher frame rate than the first CCD camera that captured the whole image of the shear band [11]. The enlarged pictures show the alternative pattern of the concentrated fringes between the dense and coarse states. It is likely that as the shear-band drifts in the flow field its velocity variation spreads out because the leading edge has the higher velocity than the tailing edge (i.e., the shear-band represents a positive charge). Consequently, the charge density represented by $\rho=$ $=\varepsilon(\partial u / \partial x+\partial v / \partial y)$ fluctuates. When $\rho$ is high, the energy dissipating effect due to the second term on the right-hand side of Eq. (5) is so large that the oscillatory characteristic decays immediately. As the shear-band enters the coarse state, $\rho$ becomes low and the oscillatory characteristic resumes. This explanation is consistent with the previous observation that the appearance of a shear-band coincides with the sharp stress drop on the stress-strain curve; when the restoring mechanism gets less effective, the stretch does not cause an increase in the stress.

\section{Conclusions}

The dynamics of plastic deformation was considered based on the restoring and energy dissipating mechanisms derived from the mesomechanical field equation. The restoring mechanism was explained as due to the medium's restoring torque driven by shear stress, and responsible for the oscillatory characteristics of the displacement field. The energy dissipating mechanism was explained as the electricforce like field force associated with the electric-charge like quantity, and responsible for the decaying characteristics of the displacement field. Experimental evidence was provided to support these explanations.

\section{References}

[1] Physical Mesomechanics of Heterogeneous Media and ComputerAided Design of Materials, Ed. by V.E. Panin, Cambridge International Science, Cambridge, 1998.

[2] V.E. Panin, Yu.V. Grinyaev, V.E. Egorushkin, I.L. Buchbinder, and S.N. Kul'kov, Spectrum of excited states and the rotational mechanical field, Sov. Phys. J., 30 (1987) 24.

[3] A.M. Kosevich, E.M. Lifshitz, L.D. Landau, L.P. Pitaevskii, Theory of Elasticity, Ed. by L.D. Landau and E.M. Lifshitz, Theoretical Physics, V. 7, Butterworth-Heinemann, Oxford, 1986.

[4] V.E. Egorushkin, Gauge Theory in Mechanics of Continuous Media, in Structural Level of Plastic Deformation and Fracture, Ed. by V.E. Panin, Nauka, Novosibirsk (1990) 20 (in Russian).

[5] S. Yoshida, Physical Mesomechanics as a Field Theory, Phys. Mesomech., 8, No. 5 (2005) 15.

[6] V.E. Egorushkin, Dynamics of plastic deformation, Russ. Phys. J., 35 (1992) 316.

[7] S. Yoshida, Interpretation of mesomechanical behaviors of plastic deformation based on analogy to Maxwell electromagnetic theory, Phys. Mesomech., 4, No. 3 (2001) 29.

[8] V.I. Danilov, V.E. Panin, N.M. Mnikh, L.B. Zuyev, Relaxation wave during plastic deformation of amorphous alloy $\mathrm{Fe}_{40} \mathrm{Ni}_{40} \mathrm{~B}_{20}$, Phys. Met. Metall., 69, No. 6 (1990) 181.

[9] V.I. Danilov, L.B. Zuyev, N.M. Mnikh, V.E. Panin, L.V. Shershova, Wave effects during plastic flow of polycrystalline Al, Phys. Met. Metall., 71, No. 3 (1991) 187.

[10] S. Yoshida, B. Siahaan, M.H. Pardede, N. Sijabat, H. Simangunsong, T. Simbolon, A. Kusnowo, Observation of plastic deformation wave in a tensile loaded aluminum-alloy, Phys. Lett. A, 251 (1999) 54.

[11] S. Yoshida, S. Toyooka, Field theoretical interpretation of dynamics of plastic deformation - Portevin - Le Chatelie effect and propagation of shear band, J. Phys.: Condens. Matter, 13 (2000) 6741.

[12] S. Yoshida, Optical interferometric study on deformation and fracture based on physical mesomechanics, Phys. Mesomech., 2, No. 4 (1999) 5 .

[13] J. Løkberg, Recent Developments in Video Speckle Interferometry, in Speckle Metrology, Optical Engineering, Vol. 38, Ed. by R.S. Sirohi, Marcel Dekker, New York (1993) 157.

[14] S. Toyooka, R. Widiastuti, Z. Qingchuan, H. Kato, Dynamic observation of localized strain pulsation generated in the plastic deformation process by electronic speckle pattern interferometry, Jpn J. Appl. Phys., 40 (2001) 310.

[15] S. Yoshida, H. Ishii, K. Ichinose, K. Gomi, K. Taniuchi, An optical interferometric band as an indicator of plastic deformation front, J. Appl. Mech., 72 (2005) 792.

[16] S. Yoshida, Suprapedi, R. Widiastuti, M. Pardede, S. Hutagalong, J.S. Marpaung, A.F. Muhardy, A. Kusnowo, Direct observation of developed plastic deformation and its application to nondestructive testing, Jpn J. Appl. Phys. Lett., 35 (1996) L854.

[17] S. Yoshida, I. Muhamed, M. Pardede, R. Widiastuti, Muchiar, B. Siahaan, A. Kusnowo, Optical interferometry applied to analyze deformation and fracture of aluminum alloys, Theor. Appl. Fract. Mech., 27 (1997) 85. 